\section{Teledermatoskopie spürt Hautkrebs sicher auf}

\begin{abstract}
Die Teledermatoskopie hat sich bereits mehrfach als zuverlässiges Verfahren für die Beurteilung pigmentierter Hautmale bewiesen. Unklar ist bislang, ob sie bei der Krebsfrüherkennung mit der direkten Begutachtung Schritt halten kann.
\end{abstract}

W ie es um die diagnostische Sicherheit der Teledermatoskopie beim Hautkrebsscreening im Vergleich zur direkten Beurteilung am Patienten steht, haben Dermatologen aus Österreich untersucht. Dazu haben sie 70 Patienten der dermatologischen Klinik in Graz mit einem moderaten bis hohen Melanomrisiko rekrutiert. Die Pigmentflecken der Probanden wurden zunächst vor Ort von einem erfahrenen Dermatologen dermatoskopisch beurteilt. Parallel wurden die Hautmale von einer versierten Pflegekraft dokumentiert und die Daten an vier Dermatologen mit großer Expertise bei der dermatoskopischen Befundung nach Österreich, Deutschland und Neuseeland verschickt. Die Dokumentation umfasst neben Übersichtsaufnahmen von jeder Körperregion, Nahaufnahmen sowie dermatoskopische Bilder auffälliger Male. Zur Verfügung standen schließlich 1.680 Übersichtsaufnahmen (24 pro Patient) und 1.922 Detailaufnahmen.
Der Dermatologe vor Ort sprach sich bei 20 Patienten für eine Exzision aus (n $=23$ Leberflecke). 48 Patienten gab er die Empfehlung, die Pigmentflecken monatlich selbst zu kontrollieren, und zwei Patienten, engmaschig zur Kontrolle in die Klinik zu kommen. Bei der histopathologischen Aufarbeitung stellten sich neun der 23 entfernten Male als Melanome heraus, zwölf wurden als melanozytäre Nävi mit atypischer Architektur identifiziert und bei zwei Proben handelte es sich um noduläre Basalzellkarzinome.

Die Experten in der Ferne beurteilen übereinstimmend den Großteil der Male $(\mathrm{n}=1.787)$ als gutartig und empfahlen die regelmäßige Kontrolle durch den $\mathrm{Pa}$ tienten. Die Notwendigkeit zur zeitnahen ärztlichen Kontrolle hingegen haben die vier Experten unterschiedlicher eingeschätzt, sie betraf 7 bis 72 Male. Zur Exzision rieten sie bei insgesamt 51 Hautmalen, wobei die Anzahl je nach Untersucher zwischen 17 und 34 lag. Bei
12 Veränderungen jedoch waren sich alle vier Experten über die Notwendigkeit der Exzision einig. Darunter waren die neun Melanome und ein Basalzellkarzinom. Insgesamt erwies sich die Interobserver-Variabilität als gering, was für eine sehr gute Übereinstimmung der diagnostischen Befunde aller fünf Dermatologen spricht („prevalence-adjusted and bias-adjusted kappa“, PABAK 0,95).

Über einen Zeitraum von vier Jahren konnten die Male von 35 Patienten regelmäßig nachkontrolliert werden. Während dieser Zeit wurde ein weiteres Mal als Melanom identifiziert, das zu Beginn als gutartig eingestuft worden war.

Fazit: Die Teledermatoskopie ist nach Ansicht der Studienautoren ein zuverlässiges Screeningverfahren zur Hautkrebsfrüherkennung und könnte alternativ oder zusätzlich zur klassischen direkten Untersuchung des Patienten eingesetzt werden. Das Verfahren erleichtere somit vor allem Risikopatienten den Zugang zu dermatoskopisch versierten Experten.

Dagmar Kraus

Arzberger E et al. Teledermoscopy in High-risk Melanoma Patients: A Comparative Study of

Face-to-face and Teledermatology Visits. Acta Derm Venereol. 2016;96:779-83

\title{
Ein Mund voll Warzen
}

Ein 36-jähriger Mann mit AIDS und Kaposi-Sarkom hatte über zwei Jahre hinweg ausgedehnte, schmerzlose papillomatöse Veränderungen in der Mundhöhle entwickelt. Er lebte in einer gleichgeschlechtlichen Lebensgemeinschaft, sein Partner berichtete anamnestisch über anogenitale Warzen. Der Patient stand unter einer antiretroviralen Therapie, seine Viruslast lag unter 20 Kopien/ml, die CD4-Zellzahl betrug 318/ml. In der gesamten Mundhöhle, am Rachen und auf der Zunge fanden sich warzenförmige Erhebungen, der typische Aspekt von Condylomata acuminata. Die Blickdiagnose wurde histologisch und immunhistochemisch bestätigt, man entdeckte humane Papillomaviren (HPV) der Typen 6 und 11. In den nachfolgenden zwei Jahren unterzog sich der Patient vielfach Lokalbehandlungen mit dem CO2-Laser, um die Läsionen entfernen zu lassen.

HPV-assoziierte Kondylome sind bei Patienten mit HIV-Infektion hartnäckiger, ausgedehnter und aggressiver als bei Patienten ohne Begleitinfektion. Eine CD4-Zellzahl unter 200/ml macht konventionelle Therapiemaßnahmen weniger effektiv und erhöht das Risiko für Rezidive.

Prof. Hermann Füeß।

Posse JL et al. Oral HPV-associated papillomatosis in AIDS. N Engl J Med. 2016;374:2585

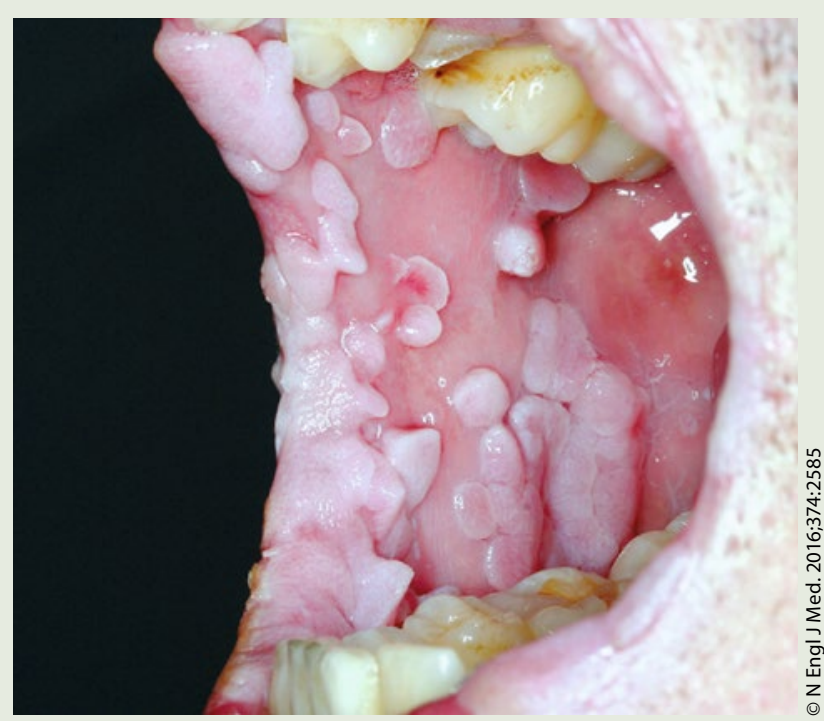

Papillomatöse Veränderungen in der Mundhöhle 